\title{
Kilovoltage Cone Beam Computed Tomography
}

National Cancer Institute

\section{Source}

National Cancer Institute. Kilovoltage Cone Beam Computed Tomography. NCI

Thesaurus. Code C104880.

Cone beam computed tomography that includes kilovoltage images. 